\title{
Jogos Sérios Competitivo-Colaborativos: Um Mapeamento Sistemático da Literatura
}

\author{
Diego Buchinger, Marcelo da Silva Hounsell \\ Programa de Pós Graduação Stricto Sensu em Computação Aplicada (PPGCA) - \\ Universidade do Estado de Santa Catarina (UDESC), Joinville - Santa Catarina - Brasil \\ dark.db@hotmail.com, marcelo@joinville.udesc.br
}

\begin{abstract}
Many studies support serious games as a tool for motivating and learning improvement. Some work have already evaluated the influence of competition and collaboration aspects on learning using such games but little research has been published regarding the usage of both aspects at the same time. A systematic literature mapping has been done, searching for serious games that have embodied both collaboration and competition in its gameplay. From 742 papers analyzed, 17 of them were selected. The mapping shows that the growth of such research topic has been erratic but increasing, there is no preferred application topic for these games and; they lack an evaluation on the learning efficacy.
\end{abstract}

Resumo. Muitos estudos apoiam o uso de jogos sérios como ferramenta para motivar e melhorar o aprendizado. Algumas pesquisas já avaliaram a influência da competição e colaboração para o aprendizado em tais jogos, mas são poucas as pesquisas sobre o uso de ambos, simultaneamente. Foi feito um mapeamento sistemático da literatura sobre jogos sérios que incorporaram tanto colaboração quanto competição em sua jogabilidade e, de uma lista de 742 artigos analisados, 17 foram selecionados. $O$ mapeamento permitiu descobrir que o crescimento dessa linha de pesquisa tem sido irregular, mas crescente; não há área de aplicação preferida e; estes tipos de jogos sérios carecem de avaliação de eficácia de aprendizagem.

\section{Introdução}

A educação nunca esteve tão relacionada ao entretenimento como está hoje: aprender e ensinar de forma lúdica se tornou objeto de pesquisa e estudos, e atualmente já é uma realidade. Foi em 2002 que se deu início ao movimento de Jogos Sérios, que se espalhou rapidamente graças à Serious Games Initiative (Susi, Johannesson e Backlund, 2007). Entende-se Jogos Sérios como aplicações que mesclam aspectos sérios como o ensino, a aprendizagem, a comunicação e a informação, com o lúdico e interativo fornecido pelos videogames, sendo o primeiro o principal objetivo e não somente o entretenimento (Michael e Chen, 2006; Alvarez e Djaouti, 2011).

Certos videogames prontos (edições comerciais COTS - Commercial Off-TheShelf) também podem ser utilizados para propósitos sérios (Alvarez e Djaouti, 2011; Squire et al., 2005). Todavia, são os Jogos Sérios que possuem o objetivo principal e inicial de servir a um propósito sério, em especial, a educação. Os jogos - sejam eles 
videogames COTS ou Jogos Sérios - podem ser classificados sob diversas perspectivas. Uma delas tem relação com a interação entre jogadores, geralmente generalizadas como: jogos para um único jogador (single player) e jogos para múltiplos jogadores (multiplayer), mas num estudo realizado por Stenros, Paavilainen e Mäyrä (2009) sobre a sociabilidade e o jogo social presentes em jogos, foi apresentada uma instância de categorização definindo cinco modalidades: único jogador, dois jogadores, múltiplos jogadores, massivo para único jogador, e massivo para múltiplos jogadores.

Alguns pesquisadores já estudaram os efeitos dos jogos com interação entre múltiplos jogadores, em especial os jogos competitivos e os colaborativos, e argumentaram que embora as pesquisas indicassem que o aprendizado colaborativo era mais efetivo do que o aprendizado individual ou competitivo, as escolas continuavam com a conduta de individualizar o processo de ensino (Johnson e Johnson, 1988). Embora os jogos competitivos encorajem os aprendizes a trabalhar mais e melhor com seus colegas, e sejam os mais comuns atualmente (Zea et al., 2009), diversas pesquisas envolvendo jogos digitais (Zea et al., 2009; Padrós, Romero e Usart, 2012; Paraskeva, Mysirlaki e Papagianni, 2010, Sung e Hwang, 2013; Buchinger, Hounsell e Dias, 2012) estão utilizando abordagens colaborativas para avaliação de aprendizagem, eficácia e motivação, obtendo bons resultados.

Considerando estas boas perspectivas que os jogos colaborativos e competitivos oferecem, mesclar tais benefícios em um só modelo de jogo sugere que haveria múltiplos benefícios. De acordo com Johnson e Johnson (1988) e Zea e colegas (2009) existem basicamente três modelos de interação para jogos: individualistas, colaborativos/cooperativos e competitivos. Todavia, pode-se pensar em um modelo de interação constituído por competição e colaboração ao mesmo tempo. Este modelo competitivo-colaborativo pode ser encontrado em videogames COTS, principalmente em jogos de estratégia, tiro e esportes, onde se formam equipes que disputam por algum objetivo que estabelece a vitória. No contexto de Jogos Sérios entretanto, esse modelo de jogo ainda não foi amplamente investigado. Com o objetivo de identificar trabalhos e pesquisas que já envolveram tal modelo em Jogos Sérios, apresenta-se e discute-se neste artigo um Mapeamento Sistemático da Literatura, realizado para evidenciar a seguinte pergunta de pesquisa: Qual o status da pesquisa em Jogos Sérios CompetitivoColaborativo, considerando os últimos 10 anos?

A fim de apresentar este mapeamento sistemático, as seções deste artigo foram escritas da seguinte forma: na segunda seção apresenta-se o processo de pesquisa bibliográfica; na terceira seção é apresentado o mapeamento sistemático; na quarta seção é apresentada uma discussão sobre os resultados obtidos; na quinta seção são apresentadas as conclusões, seguida pelos agradecimentos e lista de referências.

\section{Pesquisa Bibliográfica}

No processo de produção científica, a pesquisa bibliográfica sumariza e provém uma visão geral de uma área de conhecimento em face de seu amadurecimento. Para atingir este objetivo, os trabalhos dedicados a realizar uma pesquisa bibliográfica devem seguir metodologias sistemáticas e que podem ser categorizados de acordo com a forma de obtenção dos dados de pesquisa e apresentação dos resultados como: Mapeamento Sistemático de Literatura (SLM, Systematic Literature Mapping) - uma pesquisa em largura que categoriza os trabalhos relacionados e indica tendências, fornecendo também 
um resumo visual (Petersen et al., 2008) - e; como Revisão Sistemática de Literatura (SLR, Systematic Literature Review) - uma pesquisa em profundidade de um fenômeno de interesse que produz resultados específicos e detalhados por meio da análise de conteúdo e qualidade do material pesquisado (Kitchenham, 2004).

Neste estudo foi adotado o modelo de SLM para investigar o estado da arte do tema "Jogos Sérios Colaborativo-Competitivos", utilizando o modelo em cinco fases apresentado por Petersen et al. (2008). Na primeira fase do processo, definida como "Questão de Pesquisa", o objetivo é identificar o foco da investigação que irá delimitar o escopo da busca. Para a segunda fase, "Realização da Busca", devem ser selecionados os Mecanismos de Busca Acadêmica (MBAs) considerados relevantes para a área da Ciência da Computação e definir a sistemática para buscar todos os artigos científicos em potencial segundo uma chave de busca com palavras bem definidas. $\mathrm{Na}$ "análise de artigos", terceira fase do processo, deve-se definir critérios de inclusão e exclusão que servirão para filtrar os artigos relevantes para a questão de pesquisa do estudo. Na quarta fase do processo, "determinação das classes", identificam-se variantes de certos itens e depois aglomeram-se alguns conforme afinidade posterior. A fase "extração de dados e mapeamento" apresenta os resultados na forma de mapas e gráficos.

\section{Mapeamento Sistemático}

O objetivo inicial deste mapeamento sistemático da literatura é encontrar quaisquer trabalhos que apresentem, descrevam ou façam alguma revisão sobre Jogos Sérios Competitivo-Colaborativos, que foi alacançado conforme descrito a seguir.

\subsection{Estratégia de Busca}

Para montar a estratégia de busca desta revisão, foram selecionadas as palavras-chaves, em inglês, que definem a temática foco: (1) Serious Game, (2) Competition e (3) Collaboration. Teve-se o cuidado de incluir na frase de busca os termos variantes (e.g. competitive, competing, collaborative, collaborating) e outras flexões gramaticais de número, através de caracteres coringas, e também atenção para incluir a palavra compete, mas não outras palavras com o mesmo radical, tais como competence ou competency. A frase de busca foi então definida da seguinte forma:

$$
\text { ("serious game*”) AND (collaborat*) AND (compete OR competi*) }
$$

Foi decidido que seriam analisados apenas títulos, resumos e palavras-chaves dos artigos indexados pelos MBA e nos últimos 10 anos (2003 a 2013). Foi decidido também que seriam escolhidos apenas os documentos que foram escritos na língua inglesa ou portuguesa, e que foram publicados na forma de artigos em periódicos (journals) ou eventos (proceedings). Para a execução das buscas foram escolhidos cinco MBA consagrados na área da computação: ACM Digital Library (ACM), IEEE Xplore (IEEE), Science Direct (SD), Scopus e Web of Knowledge/Science (WoK). Apesar de cada mecanismo apresentar suas particularidades para compor a frase de busca, foi possível defini-la de acordo com o desejado.

$\mathrm{Na}$ busca inicial, feita em maio de 2013, foram encontrados poucos artigos que possuíam as palavras da frase de busca, conforme mostra a segunda coluna da Tabela 1. No total, sem considerar as ocorrências repetidas entre os mecanismos de busca, foram encontrados 26 artigos. Com uma quantidade tão reduzida de referências iniciais, que 
ainda passariam por outros critérios de análise, decidiu-se realizar uma nova pesquisa, trocando o termo Serious Game por Game, pois cogitou-se que alguns Jogos Sérios poderiam estar sendo apresentados apenas como jogos. Com esta generalização o resultado das buscas foi incrementado, ver Tabela 1, totalizando 742 artigos. Em consequência, houve um aumento significativo de artigos que atendiam a frase de busca mas num sentido divergente ao foco da pesquisa, ressaltando-se a importância da definição de critérios de inclusão e exclusão aplicados nas fases subsequentes.

Tabela 1. Documentos encontrados nas etapas iniciais do MSL.

\begin{tabular}{lcccc}
\hline Mecanismos & $\begin{array}{c}\text { Busca por } \\
\text { Serious Games }\end{array}$ & $\begin{array}{c}\text { Busca por } \\
\text { Games }\end{array}$ & $\begin{array}{c}\text { Critério de } \\
\text { Inclusão (CI-1) }\end{array}$ & $\begin{array}{c}\text { Critério de } \\
\text { Exclusão (CE-1) }\end{array}$ \\
\hline ACM DL & 3 & 37 & 19 & 19 \\
IEEE Xplore & 6 & 129 & 18 & 18 \\
Science Direct & 0 & 41 & 2 & 2 \\
Scopus & 12 & 320 & 51 & 43 \\
WoK & 5 & 215 & 26 & 22 \\
\hline Total & 26 & 742 & 116 & 104 \\
\hline
\end{tabular}

\subsection{Critérios de Inclusão e Exclusão}

Com o objetivo de filtrar apenas os artigos que possuem realmente alguma relação com a temática escolhida, foram definidos critérios de inclusão (CI) e exclusão (CE), cuja aplicação foi feita sobre a análise do título, resumo e palavras-chaves de cada artigo:

CI-1: Pelo resumo, manter o artigo que tratam aspectos competitivo-colaborativos;

CE-1: Eliminar artigos que não se tenha acesso ao texto na integra, considerando a disponibilidade fornecida pelo Portal da Coordenação de Aperfeiçoamento de Pessoal de Nivel Superior (CAPES) ou pela oferta gratuita na web;

\section{CE-2: Eliminar a duplicidade de artigos.}

$\mathrm{Na}$ Tabela 1 foram identificados os números de artigos que atenderam ao CI-1 (116 artigos) em cada MBA, assim como os documentos que passaram pelo CE-1 (104 artigos). A relação de filtragem dos artigos pelo CE-2 resultou em 50 artigos e será discutida na próxima subseção. Dessa forma, dos 742 artigos encontrados inicialmente, apenas $50(\approx 7 \%)$ foram escolhidos para uma segunda análise mais detalhada.

Efetuada a seleção dos artigos, iniciou-se a etapa de leitura e avaliação de cada um deles, através da qual se buscou descobrir quais pesquisas realmente tinham relações com Jogos Sérios, uma vez que, com a generalização para o termo Games, apareceram muitos resultados referentes a outros contextos envolvendo jogos, que não puderam ser distinguidos pelo título, resumo e palavras-chaves (CI-2). Após a aplicação deste CI, as publicações referentes a um mesmo projeto de pesquisa foram agrupadas (CE-3):

CI-2: Pelo conteúdo do artigo, manter somente aqueles que realmente tratam de um Serious Game (de acordo com a definição adotada).

CE-3: Excluir da contagem (mantendo o conteúdo) os artigos que descrevam aspectos diferentes do mesmo jogo. 
Com a aplicação do CI-2, dentre os 50 artigos, 17 foram escolhidos. Ressalta-se que dentre a lista de artigos excluídos havia quatro Massive Multiplayer Online Games (MMOG), os quais possuíam interações competitivas e colaborativas, mas que, de acordo com a definição de Stenros, Paavilainen e Mäyrä (2009), embora suportem múltiplos usuários, estariam num outro grupo de interações. Da lista de 17 artigos, aplicou-se o CE3 , que agrupou 8 artigos em 3 projetos distintos de jogos. Assim, a lista final de projetos encontrados que envolvem Jogos Sérios e interações Competitivo-Colaborativas resultou em 12 jogos distintos.

\subsection{Análise Preliminar}

Tomando os 17 artigos selecionados até o critério de inclusão CI-2, desconsiderando neste momento o agrupamento realizado pelo critério de exclusão CE-3, realizou-se uma análise da característica temporal de publicação, que se mostrou variante porém, com crescimento nos últimos 2 anos (Figura 1-a). Ressalta-se que o valor de 2013 representa um valor parcial das publicações até o primeiro bimestre do ano, considerando também o atraso na indexação de publicações recentes pelos MBAs.

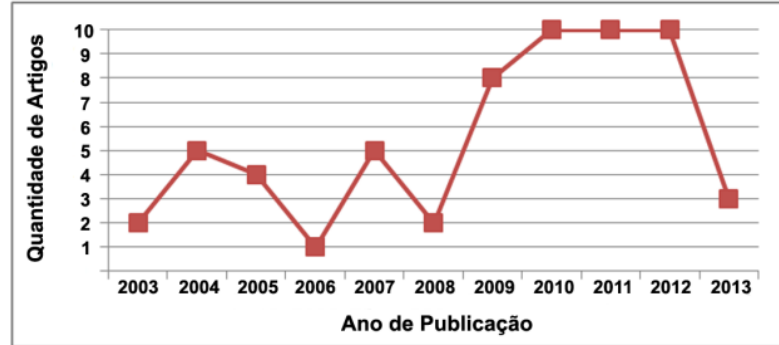

(a)

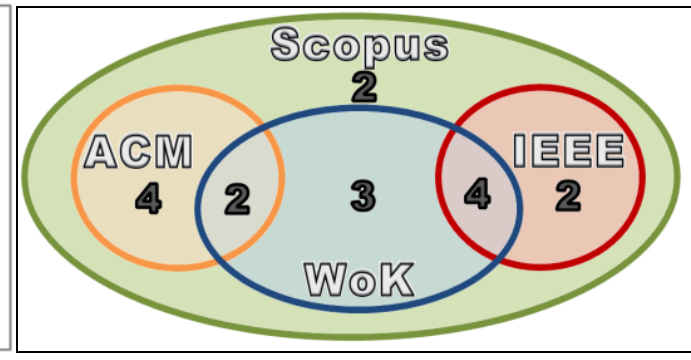

(b)

Figura 1. Publicações de Jogos Sérios competitivo-colaborativos ao longo dos anos (a), e cobertura dos artigos selecionados pelos MBAs utilizados (b).

A cobertura do conteúdo encontrado pelos MBAs utilizados também foi analisada individualmente. A fim de mostrar essa relação de cobertura foi composta a Figura 1-b apresentando um diagrama de conjuntos que relaciona os números de artigos com os MBAs utilizados. Pode-se perceber que: o MBA que indexou o maior número de resultados foi o Scopus, abrangendo 100\% dos artigos; o mecanismo WoK também apresentou boa contribuição (53\%), enquanto que ACM e IEEE apresentaram menos resultados gerais (24\% e $12 \%$, respectivamente), e SD não apresentou nenhum resultado (sendo omitido da Figura 1-b). Pode-se perceber ainda uma relação de complementaridade entre ACM e IEEE, sendo que nenhum artigo encontrado na base da ACM estava presente na base da IEEE e vice-versa.

\subsection{Coleta de Dados}

Com a lista de projetos envolvendo Jogos Sérios Competitivo-Colaborativos, foram coletados dados representativos de características genéricas dos jogos apresentados. As referências dos 17 artigos analisados para este estudo estão apresentadas no Quadro 1.

Inicialmente verificou-se a relação de apresentação como jogos, identificando a presença de três termos utilizados: Game, utilizado seis vezes (50\%); Serious Game, utilizado quatro vezes $(\approx 33,3 \%)$; e Game Based Learning Environment (GBLE), também simplificado como Learning Environment, utilizado duas vezes $(\approx 16,7 \%)$. 


\section{Quadro 1. Lista de artigos considerados na LSM.}

Bruzzone et al. (2009). Simulation for Education in Resource Management in Homeland Security;

Callaghan et al. (2010). Game-based Strategy to Teaching Electronic \& Electrical Engineering in Virtual Worlds;

Callaghan et al. (2012). Circuit Warz, the games; Collaborative and Competitive Game-based Learning in Virtual Worlds;

Callaghan et al. (2013). Using Game-Based Learning in Virtual Words to Teach Electronic and Electrical Engineering;

Chaboissier, Isenberg, e Vernier (2011). Real Time Chess: Lessons from a Participatory Design Process for a Collaborative Multi-Touch, Multi-User Game. ITS '11;

Chen et al. (2005). Animal Companions as Motivators for Teammates Helping Each Other Learn;

Gorman (2009). Serious Games, Sustainable Civilizations and Trading Zones;

Hannig (2012). eMedOffice: a Web-based Collaborative Serious Game for Teaching Optimal Design of a Medical Practice;

Hung e Young (2007). Constructing the Game-based Environment on Handheld Devices to Facilitate English Vocabulary building;
Isaacs (2008). Greening the Supply Chain: Development of a Computer Game to Teach Environmentally Benign Manufacturing;

Lin et al. (2007). Implementation of the Scrabble Game on the Mobile Devices to Increase English Vocabulary Acquisition;

Lin, Young e Hung (2008). The Game-based Constructive Learning Environmnet to Increase English Vocabulary Acquisition: Implementing a Wireless Crossword Fan-Tan Game (WiCFG as an example);

Madeira et al. (2011). LEY! Persuasive Pervasive Gaming on Domestic Energy ConsumptionAwareness;

Pareto et al. (2012). A Teachable-agent-based Game Affording Collaboration and Competition: Evaluating Math Comprehension and Motivation;

Sivak et al. (2007). Managing the Tradeoffs in the Digital Transformation of na Educational Board Game to a Computer-based Simulation;

Takaoka et al. (2012). A Development of Game-Based Learning Environment to Activate Interaction among Learners;

Won et al. (2013). How Flexible Grouping Affects the Collaborative Patterns in a Mobile-Assisted Chinese Character Learning Game?

Outra relação examinada foi a procedência do jogo apresentado no artigo: em onze títulos $(\approx 91,7 \%)$ os jogos foram produzidos em universidades ou por encomenda de pesquisadores e; uma única pesquisa $(\approx 8,3 \%)$ apenas apresentou intenção de utilizar um jogo comercial (COTS) como base para um jogo semelhante que seria produzido.

Quanto às áreas temáticas dos jogos, foram encontradas sete áreas distintas matemática, línguas, engenharia elétrica, engenharia industrial, medicina, conscientização, segurança, xadrez, e outro sem área temática bem definida. Ressalta-se que matemática e línguas foram contempladas por dois e três estudos distintos respectivamente. Para fins classificatórios, as áreas foram agrupadas em quatro: matemática (dois títulos, $\approx 16,7 \%$ ); línguas (três títulos, 25\%); engenharias (englobando engenharia elétrica e industrial, com dois títulos, $\approx 16,7 \%$ ) e; outros (englobando as demais áreas temáticas, com cinco títulos, $\approx 41,6 \%$ ).

O modo de interação adotado pelos jogos também foi investigado. Dentre todos os modelos apresentados, pôde-se categorizar três principais: o modo que mescla competição e colaboração por necessidade, no qual os indivíduos ou grupos são induzidos a colaborar ou cooperar com outros grupos para obter vantagens, mas ao mesmo tempo competem, pois só um indivíduo ou um grupo ganha o jogo; o modo de competição entre equipes, no qual existem times fixos competindo contra outros times, sendo cada um deles composto por indivíduos que colaboram ou cooperam; e o modo de interação livre, no qual os jogadores podem escolher se querem competir ou colaborar com os outros 
participantes negociando a condição de vitória de acordo com o interesse. Dentre os jogos encontrados, cinco $(\approx 41,6 \%)$ utilizaram o modelo de colaboração e competição por necessidade; quatro $(\approx 33,3 \%)$ o modelo de competição entre equipes e; três $(25 \%)$ utilizaram o modelo de interação livre.

Outro aspecto avaliado foi o número de jogadores por jogo. Foram encontrados diversos valores, sugerindo que não há um padrão mais utilizado por jogos com estas características: para o modo de interação competitivo e colaborativo simultâneo, 4 ou ' $\mathrm{n}$ ' participantes; para o modo de competição entre equipes, disputas entre equipes com 2, 4, 6 ou 'n' participantes, e; para o modo de interação livre 2 ou 'n' participantes.

A apresentação do jogo, testes, análises e comparações com outros jogos também foi averiguada e compilada com o critério de maturidade de Wazlawick (2009) e constatou-se que apenas os três primeiros níveis de maturidade foram atingidos. Apesar de certa distinção entre algumas pesquisas classificadas no Nível 2 - entre um grupo que apresentou o Jogo Sério e realizou experimentos bem definidos com o objetivo de avaliar alguma característica associada ao jogo (pré-adolescentes) e outro grupo que apenas apresentou um jogo diferente, sem realizar testes - auferiu-se: uma pesquisa $(\approx 8,3 \%)$ com nível de maturidade 1 ; dez $(\approx 83,4 \%)$ com nível de maturidade 2 e; uma $(\approx 8,3 \%)$ com nível de maturidade 3.

Também foi verificado o modelo dimensional dos jogos, encontrando: nove (75\%) deles com modelos 2D e três (25\%) com modelos 3D. Quanto aos dispositivos utilizados para os jogos, observou-se: oito $(66,7 \%)$ jogos foram produzidos para computador (incluindo máquinas usuais e tabletops), e quatro (33,3\%) para dispositivos móveis (incluindo tablets, celulares, PDAs). Os locais de publicação dos artigos escolhidos (neste caso todos os 17 artigos, desconsiderando o critério de exclusão CE3) também foram analisados. Foi encontrada apenas uma conferência 'Computer Support for Collaborative Learning' (2005 e 2007), de onde se obteve dois artigos/jogos.

\section{Discussão}

Com o baixo número de publicações que envolveram Jogos Sérios, Colaboração e Competição é difícil prever tendências de pesquisas relacionadas. Possivelmente a tendência que mais se destacou entre as pesquisas encontradas é a utilização de novos jogos desenvolvidos nas próprias universidades, ao invés da utilização de jogos COTS, para suporte às pesquisas. Das 12 pesquisas apenas uma apresentava pretensão de usar um COTS como base para o desenvolvimento de um novo jogo similar.

Observou-se que mesmo apresentando as características de Jogos Sérios, alguns autores ainda não utilizam o termo, e isso é um alerta, porque futuramente pesquisas que não se identifiquem como tal, podem não ser encontradas por outros pesquisadores e por consequência não ser citadas nem conhecidas. Com relação às áreas temáticas dos jogos, a diversidade encontrada pode estar mostrando uma tendência de abrangência de múltiplas áreas. Contudo, pode-se destacar as áreas de: línguas, matemática e engenharias, que apresentaram mais de um exemplar. Isto pode significar uma pequena tendência às áreas de ciências exatas e para o ensino de línguas.

Mesmo sendo uma interação restritiva à competição e colaboração, foram encontradas mais de uma forma de interação, diferenciadas por detalhes sutis. Estes modos de interação se apresentaram bem distribuídos pelo grupo de jogos encontrados. 
Destaque para o modelo que mescla aspectos competitivos e colaborativos por necessidade - quando há competição, mas também há necessidade de colaborar para se obter a vitória na competição - sendo identificado em cinco dos doze estudos.

Quanto à maturidade dos artigos selecionados, de acordo com os critérios de Wazlawick (2009), apesar de estar concentrada no nível 2, este resultado pode ser tomado como positivo, pois mesmo sendo classificadas neste nível, algumas pesquisas além de apresentarem o jogo (o que está sendo feito), realizaram testes para validar o funcionamento do jogo, ou a usabilidade, ou a curva de aprendizagem. Isso mostra que estes estudos estão muito próximos do nível de maturidade 3, o qual exige uma constatação de algo presumivelmente melhor.

Procurando por relações entre as áreas temáticas abordadas e os modos de interação com o grau de maturidade definido, foi plotado um gráfico de bolhas associando os termos (Figura 2) onde percebe-se certa distribuição regular das pesquisas dentre os critérios do eixo horizontal, e a maior concentração no nível central de maturidade no eixo vertical (lembrando que os níveis de maturidade 4 e 5 de (Wazlawick, 2009) foram omitidos pois não foram identificados nesta pesquisa). Esse resultado indica uma possível tendência de pesquisas do tipo "apresentação de algo diferente" sem realização de testes e análises comparativas, com boa distribuição nas temáticas de aplicação e nos modelos de interação escolhidos. Contudo, o número de artigos encontrados é baixo, o que dificulta a verificação de tendências.

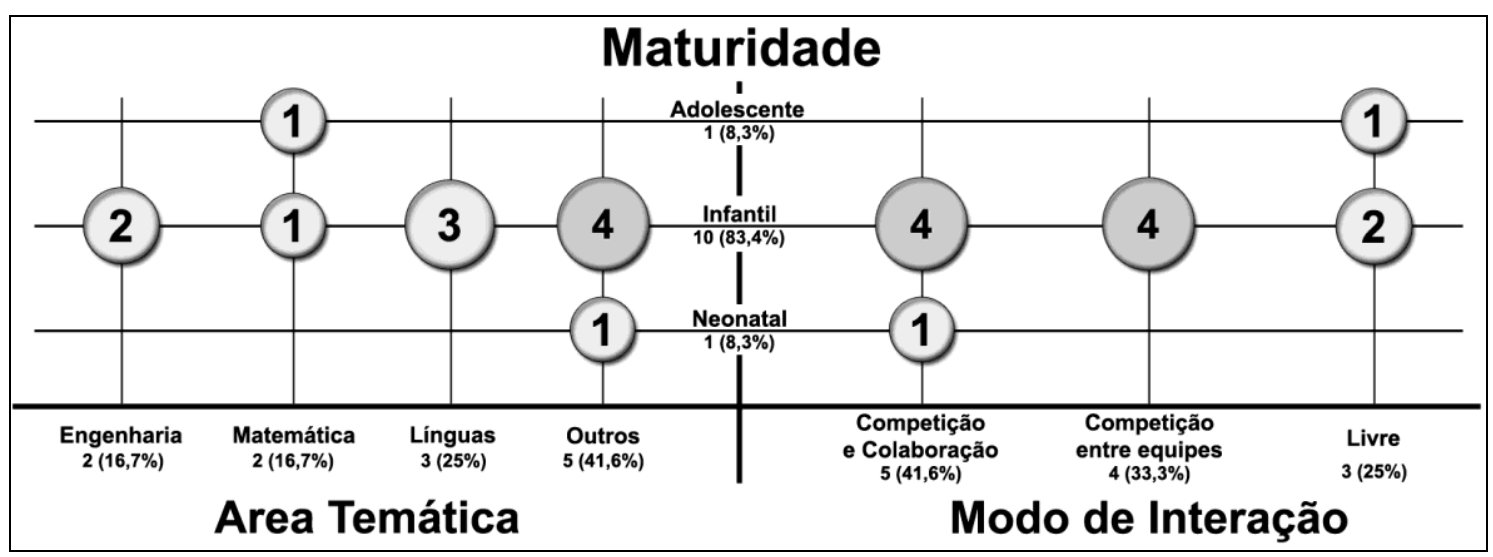

Figura 2. Resultado do mapeamento sistemático de literatura em relação à maturidade, área temática e modo de interação.

A preferência por computador como dispositivo reforça os achados de (Connolly et al., 2012), os quais evidenciaram os PCs como a plataforma mais utilizada para os jogos computacionais (computer games) e Jogos Sérios (serious games). Entretanto, os jogos voltados para dispositivos móveis foram mais contemplados com os critérios aplicados nesta pesquisa, do que na pesquisa de (Connolly et al., 2012), na qual foram encontrados apenas três artigos (de 129) voltados para esta plataforma.

A preferência por jogos em 2D possivelmente tem base na maior facilidade de criação e modelagem dos elementos do jogo e por requisitos de hardware, visto que a maioria dos dispositivos móveis ainda não suportam satisfatoriamente muitos objetos $3 \mathrm{D}$ em uma cena. Os resultados mostraram também que os Jogos Sérios CompetitivoColaborativos ainda não possuem um veículo de publicação preferencial, o que acaba dificultando encontrar e desenvolver pesquisas relacionadas. 
Por fim, na leitura detalhada de cada um dos trabalhos observou-se que em nenhum dos casos os autores mencionaram usar e nem apresentaram os jogos seguindo alguma metodologia ou guideline de concepção e design, sugerindo que não existe ainda uma metodologia estabelecida para desenvolvimeto destes tipos de jogos.

\section{Conclusão}

Com a emergência dos Jogos Sérios, emergiram também as variantes para múltiplos jogadores que contemplam os aspectos colaborativos e competitivos. Entretanto, a composição destes aspectos é uma possibilidade que pode trazer o benefício de ambas contribuindo para a motivação, maior socialização e aprendizado.

Apesar de terem sido encontrados 742 artigos com publicações sobre jogos que envolvessem competição e colaboração, apenas 17 continham realmente elementos de Jogos Sérios Competitivo-Colaborativos, apresentando somente 12 jogos - o que demonstra que este tópico ainda foi pouco investigado - e que a maioria nem se apresentava com a terminologia de Jogos Sérios. Por outro lado, constatou-se que houve uma oscilação significativa no número de publicações mas, um aumento e tendência de crescimento nos últimos anos. Apesar de Connolly (2012) ter indicado o MBA Science Direct como sendo o que mais continha publicações relativas à computer games e serious games, para o contexto de Jogos Sérios Competitivo-Colaborativos, este MBA foi o que menos contribuiu, enquanto que o Scopus foi o que mais contribuiu.

A pequena quantidade Jogos Sérios com aspectos competitivos e colaborativos juntos, se concentrou em pesquisas no nível 2 (de um total de 5) de maturidade científica (Wazlawick, 2009), o que mostra que existe carência de pesquisas em níveis mais maduros. Constatou-se ainda diversidade quanto à área temática, onde o ensino de línguas se destacou levemente mas, várias outras temáticas também foram encontradas. A maioria delas voltadas para PC e em 2D.

O que se conclui desse mapeamento sistemático é que a pesquisa em Jogos Sérios Competitivo-Colaborativos (conjuntamente) vem crescendo mas, precisa se aprofundar bastante para se ter clareza sobre os benefícios dessa vertente (colaboração e competição juntas) principalmente pelo fato das pesquisas estarem em um nível inicial de maturidade. Também ficou evidente a ausência de Metodologias voltadas especialmente para Jogos Sérios Competitivo-Colaborativos. E, essa responsabilidade parece ser da academia, uma vez que a ampla maioria destes Jogos Sérios observados foi produto de pesquisas em universidades.

\section{Agradecimentos}

Os autores gostariam de agradecer à UDESC pela bolsa de mestrado do PROMOP.

\section{Referências}

Alvarez, J., e Djaouti, D. (2011). An introduction to Serious Game Definitions and Concepts. In Proceedings of the Serious Games \& Simulation for Risks Management Workshop, p. 11-15. 
Buchinger, D., Hounsell, M. da S., e Dias, C. (2012). Colaboratividade em um Jogo Eletrônico para Ensino sobre Dengue. In $23^{\circ}$ Simpósio Brasileiro de Informática na Educação SBIE 2012, Rio de Janeiro, p. 1-10.

Connolly, T. M., Boyle, E. A., MacArthur, E., Hainey, T., e Boyle, J. M. (2012). A systematic literature review of empirical evidence on computer games and serious games. In Computers \& Education, 59, p. 661-686.

Johnson, R. T., e Johnson, D. (1988). Cooperative Learning: Two heads learn better than one, Transforming Education: In Context, \#18, Winter 1988, 34.

Kitchenham, B. (2004) Procedures for performing systematic reviews. Technical Report Technical Report TR/SE-0401, Keele University and NICTA.

Michael, D. R. e Chen, S. L. (2006). Serious Games: Games That Educate, Train and Inform. Thomson Course Technology, Boston, MA, 287p.

Padrós, A., Romero, M., e Usart, M. (2012). Measuring the knowledge convergence process in the collaborative game MetaVals. In Procedia Computer Science, p. 193202.

Paraskeva, F., Mysirlaki, S., e Papagianni, A. (2010). Multiplayer online games as educational tools: Facing new challenges in learning. In Computers \& Education, p. 498-505.

Petersen, K., Feldt, R., Mujtaba, S., e Mattsson, M. (2008). Systematic Mapping Studies in Software Engineering. In 12th International Conference on Evaluation and Assessment in Software Engineering (EASE), Italy, 10p.

Squire, K., Giovanetto, L., Devane, B., e Durga, S. (2005). From Users to Designers: Building a Self-Organizing Game-Based Learning Environment, In TechTrends, 49(5):34-42.

Stenros, J., Paavilainen, J., e Mäyrä, F. (2009). The Many Faces of Sociability and Social Play in Games. In MindTrek 2009, Tampere, Finland, ACM, p. 82-89.

Sung, H. Y., e Hwang, G. J. (2013). A collaborative game-based learning approach to improving students' learning performance in science courses. In Computers \& Education, 63, p. 43-51. DOI= http://dx.doi.org/10.1016/j.compedu.2012.11.019.

Susi, T., Johannesson, M., e Backlund, P. (2007). Serious Games - An Overview. Technical Report HS-IKI-TR-07-001, p. 1-28.

Wazlawick, R. S. (2009). Metodologia de Pesquisa para Ciência da Computação. Editora Campus, Elsevier, 1. ed. 184p.

Zea, N. P., Sánchez, J. L. G., Gutiérrez, F. L., Cabrera, M. J., e Paderewski, P. (2009). Design of education multiplayer videogames: A vision from collaborative learning. In Advances in Engineering Software, p. 1251-1260. 